\title{
FUNCTIONAL ENDOSCOPIC TRANSNASAL DACRYOCYSTORHINOSTOMY
}

\author{
H. B. WHITTET ${ }^{1}$, G. ADRIEN SHUN-SHIN ${ }^{2}$ and P. AWDRY ${ }^{2}$ \\ Oxford
}

\begin{abstract}
SUMMARY
Endoscopic nasal surgical techniques are revolutionising the management of nasal and sinus disorders. Historically the surgical approach to the nasolacrimal sac and duct has been indirect by an external approach because of poor visualisation intranasally. The nasolacrimal apparatus is, however, intimately related to the lateral nasal wall and may readily be approached using an endoscopic technique which minimises functional interference with the physiological action of the lacrimal pump. This paper describes a technique of endoscopic intranasal dacryocystorhinostomy which has success rates comparable to those obtained by the more conventional external approach.
\end{abstract}

Surgery to correct distal nasolacrimal sac and duct obstruction is commonly performed by an external approach through a skin incision. ${ }^{1}$ The traditional external approaches, whether performed with or without mucosal flap anastomoses, have a reported success rate of $85-97 \% \%^{2,3}$ dependent upon the surgeon's experience. For the most part these approaches take little account of predisposing or coexistent intranasal disorders or anatomical variants that could compromise the final surgical result.

Probing, irrigation, dilatation and intubation of the nasolacrimal duct were practised in the eighteenth and early nineteenth centuries. ${ }^{1}$ Caldwell ${ }^{4}$ and West ${ }^{5}$ described techniques for an intranasal approach to the nasolacrimal apparatus but surgical visualisation through the ipsilateral nostril was limited by the obliquity of the approach. Improved visualisation was obtained by using a transseptal approach, ${ }^{6}$ but all of these early intranasal techniques were hampered by inadequate illumination of the operative site and the external approach became the operation of choice. ${ }^{6}$ The advent of the operating microscope awakened further but sporadic otolaryngological interest in the more direct nasal approach with reported success

From: 'Departments of Otolaryngology, Radcliffe Infirmary, Oxford, and Royal Berkshire Hospital, Reading; ${ }^{2}$ Oxford Eye Hospital, Oxford, UK.

Correspondence to: Mr. H. B. Whittet, ENT Department, Royal Berkshire Hospital, London Road, Reading, Berkshire RG1 5AN, UK. rates similar to those for the external route. ${ }^{7}$ The external approach now commonly practised by ophthalmologists was first described by Toti in $1904^{8}$ in which a simple rhinostomal communication was created between the mucosal surfaces of the nasolacrimal sac and nostril. A more formal mucosal anastomosis with accurate suturing of mucosal flaps was subsequently described by DupreyDutemps and Bourget. ${ }^{9}$

The increasing use of endoscopic techniques for performing functional intranasal and sinus surgery has allowed unrivalled visualisation of the nasal cavity ${ }^{10}$ and reawakened interest in the transnasal approach to the nasolacrimal apparatus. ${ }^{11}$ An endoscopic approach has been recommended as a revision procedure for cases of failed external dacryocystorhinostomy ${ }^{12}$ and a similar approach with laser assistance and video control used as the primary surgical procedure. ${ }^{13}$

This paper describes an endoscopic intranasal approach for performing a dacryocystorhinostomy as the initial surgical approach in a prospective series of 19 patients. It emphasises the value of pre-operative CT dacryocystography in revealing anatomical variants and coexistent sinus disease that may conveniently be corrected either at the same operation or at an earlier stage.

\section{METHODS}

Nineteen patients were referred after an initial ophthalmological assessment with a diagnosis of distal nasolacrimal sac or duct obstruction. There were 8 women and 11 men with an age range of $14-80$ years (mean age 40 years). The clinical presentation was epiphora in 15 cases, recurrent dacrocystitis in 3 cases and acute nasolacrimal sac abscess in 1 case.

Predisposing causes were the consequences of previous surgery, namely intranasal antrostomies (3 cases) and lateral rhinotomy ( 1 case). One of these cases had undergone an unsuccessful external dacrocystorhinostomy some 12 months previously. The remaining cases could be grouped into an idiopathic classification. One of these cases had bilateral symptoms and had experienced a successful external procedure on the first side. 


\section{Pre-Operative Assessment}

Endoscopic. All patients were examined using $0^{\circ}$ and $30^{\circ}$ Hopkins rod telescopes to assess the anatomical accessibility of the operative site. Particular attention was paid to the presence of significant deviations of the nasal septum at the level of the middle meatus or at the anterior end of the inferior turbinate as they would unnecessarily impede endoscopic surgical access. The finding of such variants allowed adequate planning for appropriate septal surgery at the time of operation. The presence of otherwise unsuspected coexistent sinus disease could also be determined by an examination of the middle meatus in a manner well familiar to nasal endoscopic surgeons. ${ }^{14}$ This allowed for pre-operative management of such conditions as polyposis or inflammatory conditions, and forewarned the surgeon of the potential need for endoscopic ethmoidal or middle meatal surgery.

Radiological. All the referred patients had had conventional dacryocystography performed as part of their initial diagnostic ophthalmological assessment. In addition, as commonly practised in functional endoscopic sinus surgery, all the patients in this series had radiological assessment performed by CT scanning. ${ }^{15}$ Performed in both coronal and axial planes this allowed for a complete assessment of the paranasal sinuses and in particular anatomical abnormalities of the middle meatus such as a pneumatised middle turbinate anomaly (concha bullosa), or unduly extensive anterior ethmoid or agger nasi air cells that could impede access. In particular this combined approach of endoscopy and radiological assessment enabled the presence of a predisposing neoplastic lesion to be excluded. The technique of CT dacrocystography (CTDCG $)^{16,17}$ introduces contrast into the nasolacriminal sac allowing a precise level for the obstruction within the nasolacrimal sac and duct to be determined. More importantly it clearly displays the presence of potential surgical obstacles to the endoscopic approach. Of particular significance is the thickness of bone cover given by the frontal process of maxilla (Fig. 1) which would necessitate removal by drill, chisel or punch forceps at the time of surgery.

\section{Operative Technique}

The operation was performed under general anaesthesia in all cases, following nasal mucosal preparation with a $10 \%$ cocaine and 1 in 1000 adrenaline or $1 \%$ ephedrine solution. Local infiltration with 1 in 80000 adrenaline and $2 \%$ xylocaine was performed in the region of the frontal process of maxilla and superior attachment of the middle turbinate. This facilitates initial elevation of mucosal flaps by minimising mucosal bleeding. At this stage any septal deviation intruding upon the proposed operative site was corrected by a conventional submucous resection, utilising a contralateral hemitransfixion incision to minimise bleeding into the homolateral nostril.

The key initial landmark is the posterior border of the frontal process of maxilla, which is usually identifiable as an indentation into the nasal airway just anterior to the middle turbinate. A mucoperiosteal flap is elevated from the lateral nasal wall in this region centred just in front of the anterior border of the middle turbinate which demarcates the position of the nasolacrimal sac. The nasolacrimal sac and duct are often easily visible at this point covered by the thin lacrimal bone and confirmation may easily be made by gentle instrumental probing. However, removal of a prominent or overhanging frontal process of maxilla may be necessary for adequate exposure. This can be achieved by using a drill of the type used by otolaryngologists for mastoid and middle ear surgery, or by the use of a hammer and gouge and rongeurs. The lacrimal bone may now be elevated and removed and should the nasolacrimal apparatus lie more posteriorly under cover of the middle turbinate a limited anterior resection of this structure may be necessary. At this point a Liebrich lacrimal probe is inserted into the inferior canaliculus and directed against the medial wall of the lacrimal sac in order to 'tent' it intranasally. This procedure may reveal the need for further bone removal, especially at the junction of attachment of the middle turbinate and lateral nasal wall, a point that demarcates the floor of the lacrimal fossa. Inadequate bony removal at this point is a common cause of failure when performing an external dacrocystorhinostomy. ${ }^{3}$ The tented mucosa of the nasolacrimal sac is incised either by a sickle knife or by using a carbon dioxide laser to create a large mucosal window (Fig. 2). Granulations, mucopus, residual contrast material and a thickened mucosal lining may hamper identification of the common canalicular opening, but probing of both canaliculi usually confirms the positioning of the rhinostoma. The authors use O'Donoghue silastic tubing to act as a temporary stent as well as providing a means for securing a thin silastic funnel in position to maintain rhinostomal patency. Nasal packing is avoided if possible, but if necessary a non-adherent dressing may be used and is removed a few hours post-operatively.

As with endoscopic sinus surgery, close post-operative management is important to remove crusting, granulations and secretions from the rhinostoma and stents. The authors practise assessment 1 and 2 weeks post-operatively at which time fluorescein may be instilled into the conjunctival sac to assess rhinostomal patency (Fig. 3). Should this be satisfactory the patient is then reviewed at 3 months when the nasolacrimal tubing and nasal stents are removed.

\section{RESULTS}

Eighteen of the 19 procedures $(94.7 \%)$ resulted in a complete resolution of the presenting symptoms over a follow-up period that ranged from 9 to 15 months. Sixteen patients were followed up for more than 12 months. The remaining case showed an improvement in presenting epiphora although the patient complained of watering when walking in cold conditions. Scarring between the middle turbinate and lateral nasal wall did occur in 5 of the successful cases but did not interfere with the final outcome. 


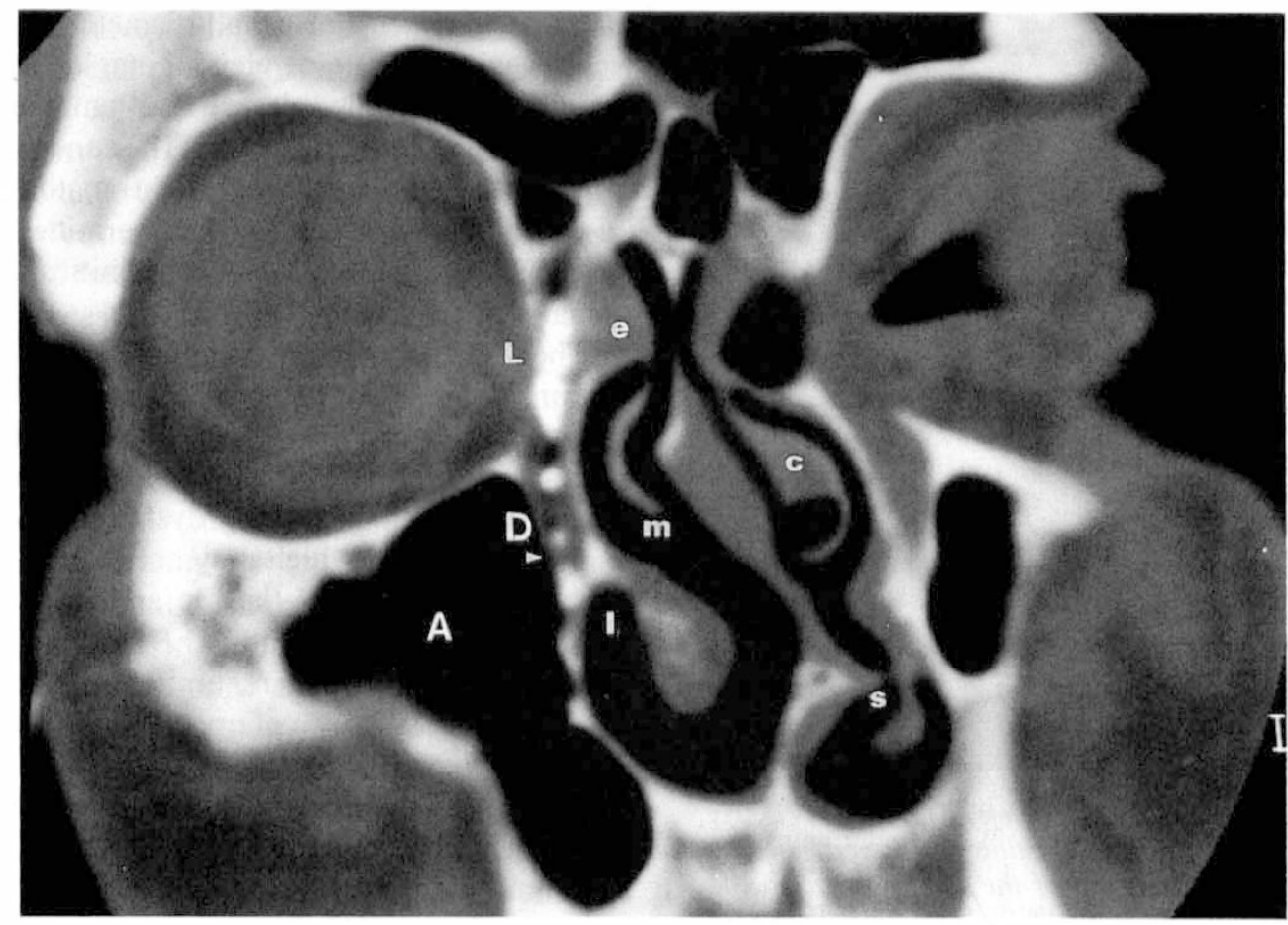

Fig. 1 a.

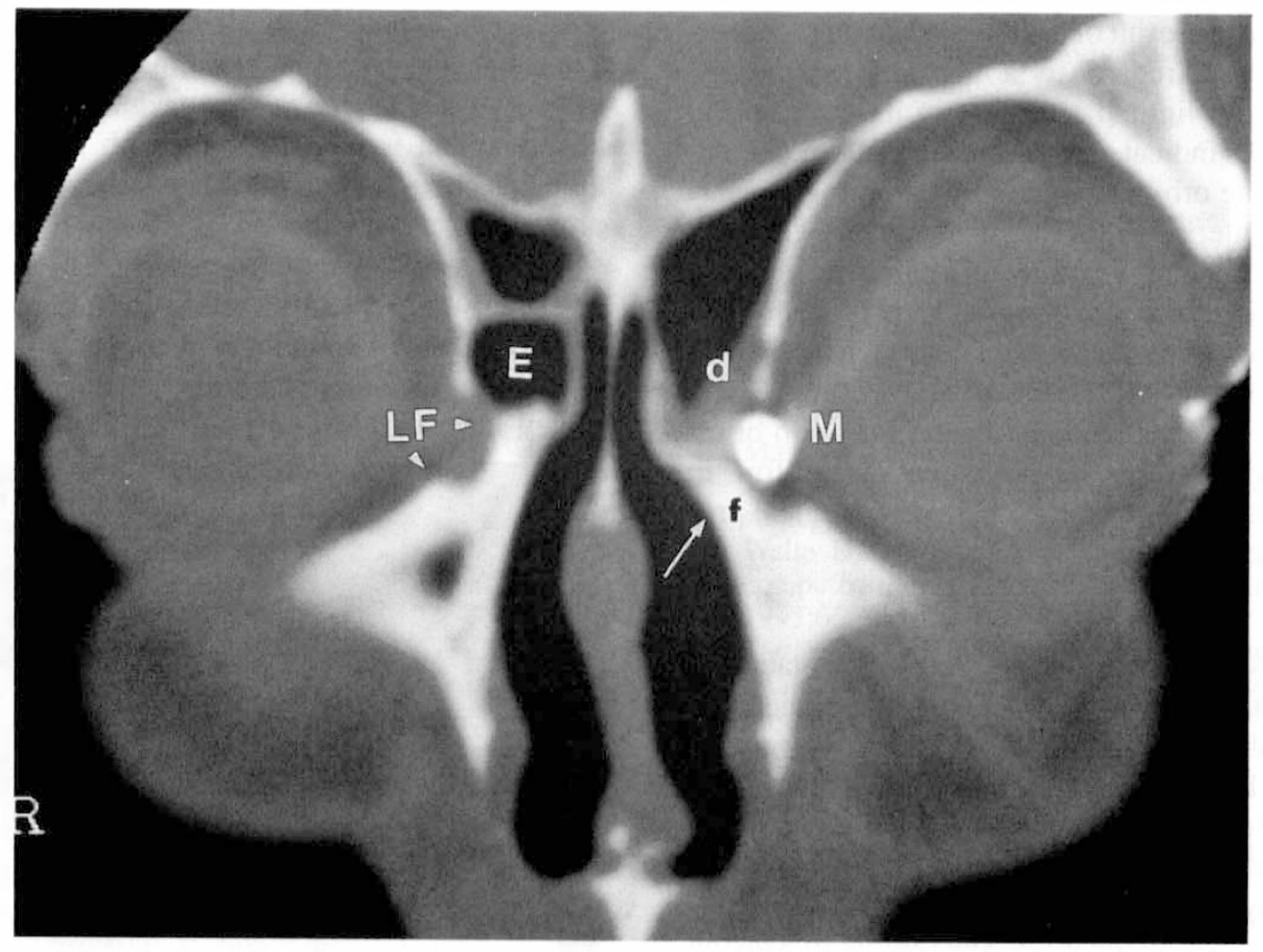

Fig. 1b.

Fig. 1. (a) A right coronal CT dacryocystogram (CT DCG) showing contrast within the nasolacrimal sac $(L)$ and duct $(D)$ up to its intranasal opening in the inferior meatus (I). Its proximity to the maxillary sinus $(A)$ and vulnerability to antral disease is highlighted (arrowhead). Coexistent anatomical nasal abnormalities are conveniently displayed: $M$, paradoxical curvature of the middle turbinate; $S$, nasal septal deviation; $C$, a pneumatised middle turbinate (concha bullosa). Assocated anterior ethmoid mucosal disease can be seen (E). (b) Coronal CT DCG of a left-sided nasolacrimal mucocoel $(M)$ clearly displayed within the bony lacrimal fossa (LF). Its intimate relationship with the frontal process of maxilla $(f)$ can be seen. The endoscopic transnasal approach is arrowed. The anterior part of the ethmoid labyrinth is closely related to the nasolacrimal apparatus (E). Secondary ethmoidal mucosal disease can be seen on the affected side $(d)$. 


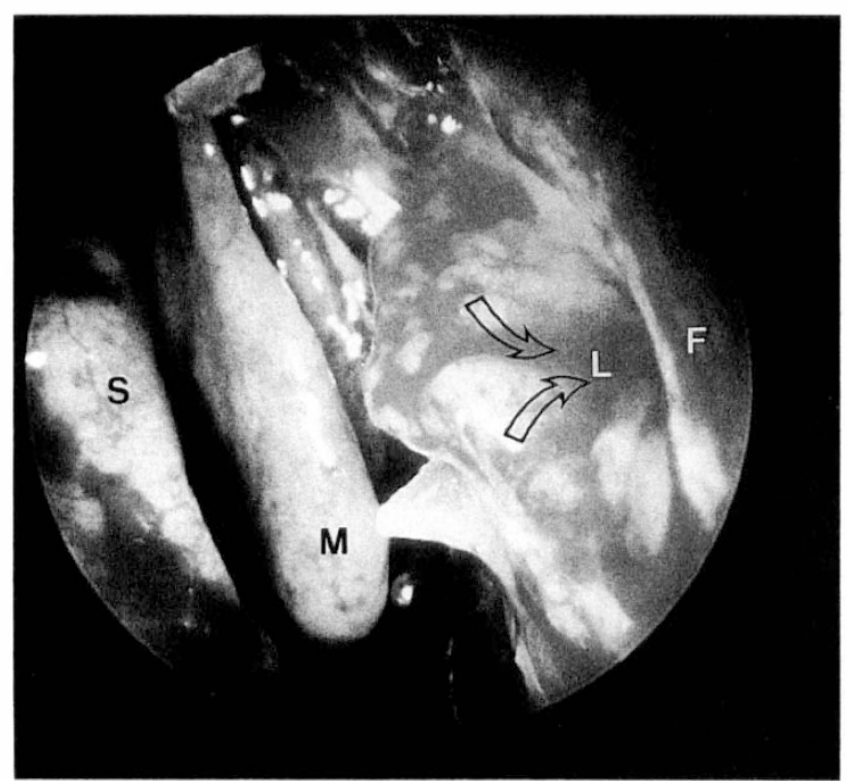

Fig. 2. Intranasal endoscopic view of left nasal airway clearly displaying a large rhinostomal window (arrowed) in the lacrimal sac $(L)$. The relationship of the lacrimal sac to the anterior end of the middle turbinate $(M)$ can be seen. $F$, frontal process of maxilla; $S$, nasal septum with incision from simultaneous submucous resection.

This seemed to be the result of an attempt at overly conservative surgery with regard to a partially obstructing middle turbinate. A limited resection of its anterior end would appear to be indicated in such circumstances. Inadvertent entry to the orbit is avoided by confining the surgical approach anterior to the lacrimal bone, well away from the ethmoid complex and lamina papyracea.

Apart from scarring occurring at the level of the middle turbinate, there were no specific complications of the procedure. Specific complications associated with the external approach such as haematoma, wound infection or air reflux did not occur in this series. Patient discomfort, in common with that in other endoscopic nasal surgical procedures, was minimal.

\section{DISCUSSION}

The variety of surgical approaches to the nasolacrimal apparatus reflects the respective viewpoints of the ophthalmologist and otolaryngologist. Nasolacrimal surgery has traditionally been the province of the ophthalmologist, especially when dealing with canalicular and punctal disorders. However, problems relating to the nasolacrimal sac and duct may be directly related to pathological processes originating within the nose. The rapidly increasing use of endoscopic techniques for diagnosis and surgical management by otolaryngologists is changing the more traditional external surgical approaches to the nose and paranasal sinuses. These new methods are based on a better understanding of the physiological and pathophysiological processes involved in nasal and sinus disease. ${ }^{18}$ The principles of endoscopic sinus surgery are minimalist, that is to perform limited but functional surgery upon areas primarily responsible for the presenting symptoms.
The development of small-diameter endoscopic telescopes with a range of viewing angles has introduced a previously unrivalled degree of illumination and visualisation of intranasal structures. The concomitant use of CT imaging in this area to highlight anatomical detail and areas of localised disease has permitted precise minisurgical procedures that provide significantly better symptomatic improvement. ${ }^{12}$

The application of these endoscopic principles to nasolacrimal sac obstruction is logical since the surgical approach depends upon the intimate relationship of this structure to the lateral nasal wall. The medial approach runs less risk of producing dysfunction of the lacrimal pump mechanism which may occur from scarring of the mobile lateral wall of the sac and the attached muscle fibres of orbicularis oculi. The fact that none of these patients complained of regurgitation of air during nose blowing, a relatively common complaint after conventional dacrocystorhinostomy surgery, attests to the preservation of the normal proximal anatomy of the lacrimal sac. Coexistent and potentially predisposing septal deflections, middle turbinate anatomical variants ${ }^{19}$ and middle meatal and ethmoid inflammatory disease states may conveniently be managed at the same procedure.

Further advantages of an intranasal approach include the lack of a potentially disfiguring scar, minimal postoperative discomfort and minimal blood loss. In contrast to the external approach the attachment of the medial canthal ligament is not disturbed with this intranasal technique. Although general anaesthesia was used for all the procedures performed in this series the ease of the approach would allow the use of local anaesthetic techniques as are frequently used in endoscopic sinus surgery. Given the lack of immediate post-operative complications there is an obvious potential application of the technique in day case surgery.

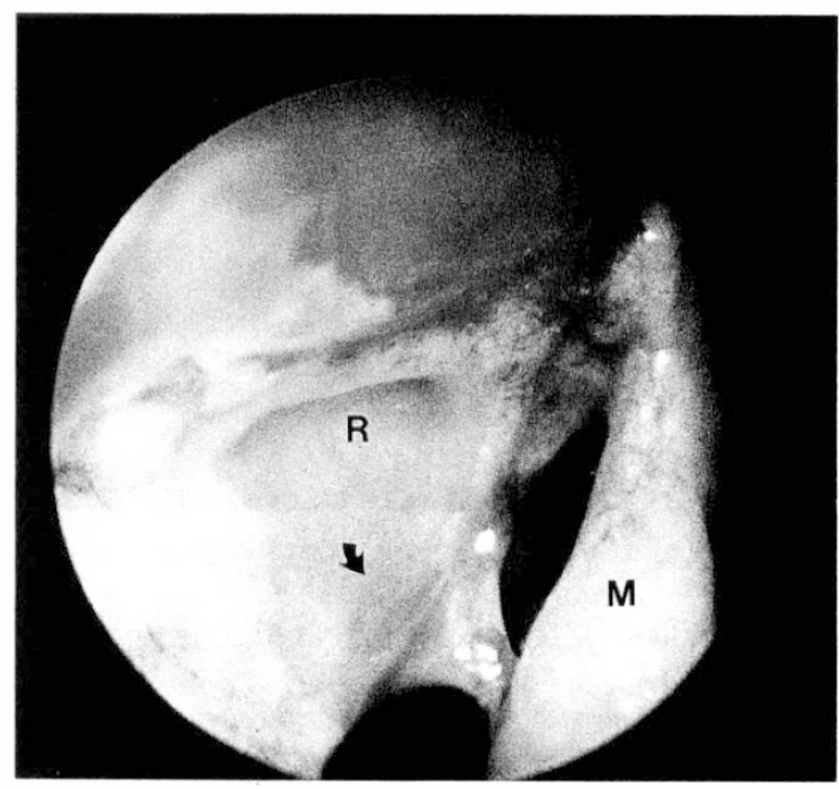

Fig. 3. Endoscopic intranasal view showing fluoroscein (arrowed) passing into the nose from a patent left rhinostome $(R) . M$, middle turbinate. 
Although not a prerequisite, radiological assessment by CT-DCG is an integral part of this approach as practised by the authors. It allows a detailed assessment of the intranasal relationships of the nasolacrimal apparatus ${ }^{20}$ and has the important side benefit of demonstrating otherwise unsuspected ethmoid sinus disease which may therefore readily be addressed at the same operation or at an earlier stage if necessary by an ENT surgeon. The technique of CT-DCG has previously been used in the evaluation of conventional dacrocystorhinostomy failures to good effect ${ }^{16}$ by revealing information that facilitated subsequent external revision surgery. Its primary use in this surgical approach was invaluable for pre-operative assessment and surgical planning.

The endoscopic nasal instrumentation used is now widely available. A basic set for this procedure would include a $0^{\circ} 4 \mathrm{~mm}$ endoscope and a handful of instruments. Assuming availability of a suitable light source and cable the total cost of this would be approximately $£ 2000$. Many of these instruments would, however, be available from otolaryngological colleagues. As with any new surgical technique there is a learning curve involved. The principal obstacle for the ophthalmologist is obtaining familiarity with the intranasal anatomy relating to the nasolacrimal apparatus as well as adapting to the use of monocular vision with the nasal endoscopes. Both difficulties may easily be overcome by initial familiarisation either by cadaveric dissection or under the guidance of an ENT colleague.

Although primarily a rhinological approach the procedure as practised by the authors has been a collaborative one. The technique involves a simple surgical dissection and therefore does not require the use of expensive and specialised laser equipment. In comparison with endoscopic sinus surgery the technical approach is straightforward with minimal risk of damage to the orbit or other vital structures and is therefore well within the province of the interested ophthalmologist and nasal endoscopic surgeon. The surgical results compare favourably with the best that can be obtained with an external approach, suggesting that this technique is an acceptable and functional alternative.

The authors would like to thank Mr. A. P. Freeland, Mr. C. A. Milford, Mr. B. Colman, Mr. W. S. Lund, Mr. T. Heyworth, Mr. R. Parker and Mr. N. Marks, Consultant ENT surgeons, Radcliffe Infimary, Oxford, and Royal Berkshire Hospital, Reading, and Mr. D. Welham, Consultant Ophthalmologist, Royal Berkshire Hospital, Reading, for their support and advice. They also acknowledge the help of Dr. D. Waite, Consultant Radiologist, Royal Berkshire Hospital, Reading, for radiology and the
Medical Illustration Department, Royal Berkshire Hospital, Reading.

Key words: Dacryocystorhinostomy, Endoscopy, Epiphora, Nasal.

\section{REFERENCES}

1. Roper-Hall MJ, editor. Stallard's eye surgery. Bristol: Wright, 1989:149-56.

2. Becker BB. Dacryocystorhinostomy without flaps. Ophthalmic Surg 1988;19:419-27.

3. Welham RA. Management of unsuccessful lacrimal surgery. Br J Ophthalmol 1987;71:152-7.

4. Caldwell GW. Two new operations for obstruction of the nasal duct with preservation of the canaliculi and an incidental description of a new lachrymal probe. NY Med J 1983;57:581.

5. West JM. A window resection of the nasal duct in cases of stenosis. Trans Am Ophthalmol Soc 1914;12:654.

6. Macbeth R. Problems of lacrimal obstruction: the rhinological approach. Trans Ophthalmol Soc 1956;LXXVI:355-68.

7. Jokinen K, Karja J. Endonasal dacryocystorhinostomy. Årch Otolaryngol 1974;100:41-4.

8. Toti . Nuovo metodo conservatore di cura radicale delle suppurazioni cronici del sacco lacrimale (dacriocistorinostomia). Clin Med Firenze 1904;10:385-7.

9. Duprey-Dutemp L, Bourguet $\mathbf{M}$. Procède plastique de dacryoscystorhinostomie. Ann Ocul 1920;158:241.

10. Wigand ME. Endoscopic surgery of the paranasal sinuses and anterior skull base. New York: Thieme, 1990.

11. McDonogh M, Meiring JH. Endoscopic transnasal dacryocystorhinostomy. J Laryngol Otol 1989;103:585-7.

12. Metson R. Endoscopic surgery for lacrimal obstruction. Otolaryngol Head Neck Surg 1991;104:473-9.

13. Gonnering RS, Lyon DB, Fisher JC. Endoscopic laserassisted lacrimal surgery. Am J Ophthalmol 1991;111: 152-7.

14. Stammberger H. Endoscopic endonasal surgery: concepts in treatment of recurring rhinosinusitis. II. Surgical technique. Otolaryngol Head Neck Surg 1986;94:147-56.

15. Zinnreich SJ, Kennedy DW, Rosenbaum AE, Gaylor BW, Kumar AJ, Stammberger H. Paranasal sinuses: CT imaging requirements for endoscopic surgery. Radiology 1987;163: 769-75.

16. Massoud TF, Whittet HB, Anslow P. CT dacryocystography for nasolacrimal duct obstruction following paranasal sinus surgery. Br J Radiol 1993;66:223-7.

17. Walte DW, Whittet HB, Shun-Shin GA. Technical note: computed tomography dacryocystography. $\mathrm{Br} \mathrm{J}$ Radiol 1993:66 (in press).

18. Stammberger $\mathrm{H}$. Endoscopic endonasal surgery: concepts in treatment of recurring rhinosinusitis. I. Anatomic and pathophysiologic considerations. Otolaryngol Head Neck Surg 1986;94:143-6.

19. Zinreich SJ, Mattox DE, Kennedy DW, Chisholm HL, Diffley DM, Rosenbaum AE. Concha bullosa: CT evaluation. J Comput Assist Tomogr 1988;12:778-84.

20. Blaylock WK, Moore CA, Linberg JV. Anterior ethmoid anatomy facilitates dacryocystorhinostomy. Arch Ophthalmol 1990;108:1774-7. 